\title{
Optimal Harvesting Strategy Based on Rearrangements of Functions*
}

\author{
Behrouz Emamizadeh $^{\dagger} \quad$ Amin Farjudian ${ }^{\dagger \S} \quad$ Yichen Liu $^{\mathbb{q}}$
}

\begin{abstract}
We study the problem of optimal harvesting of a marine species in a bounded domain, with the aim of minimizing harm to the species, under the general assumption that the fishing boats have different capacities. This is a generalization of a result of Kurata and Shi, in which the boats were assumed to have the same maximum harvesting capacity. For this generalization, we need a completely different approach. As such, we use the theory of rearrangements of functions. We prove existence of solutions, and obtain an optimality condition which indicates that the more aggressive harvesting must be pushed towards the boundary of the domain. Furthermore, we prove that radial and Steiner symmetries of the domain are preserved by the solutions. We will also devise an algorithm for numerical solution of the problem, and present the results of some numerical experiments.
\end{abstract}

Keywords: Population biology, Rearrangements of functions, Reaction-diffusion, Optimization, Symmetry.

Mathematics Subject Classification: 35K57, 35Q92, 35J25, 65K10, 65K15

\section{Contents}

$\begin{array}{lll}1 & \text { Introduction } & \mathbf{2}\end{array}$

1.1 Our alternative approach through rearrangements of functions $\ldots \ldots \ldots \ldots$

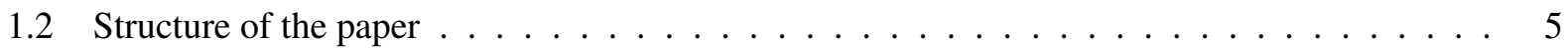

2 Preliminaries $\quad 6$

2.1 Rearrangements of functions $\ldots \ldots \ldots \ldots \ldots \ldots$

2.2 Steady state equation $60 \ldots \ldots \ldots \ldots \ldots \ldots$

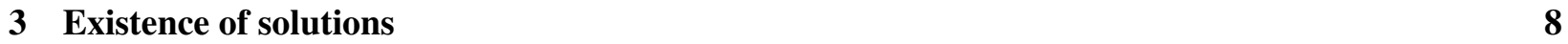

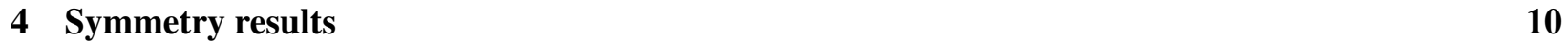

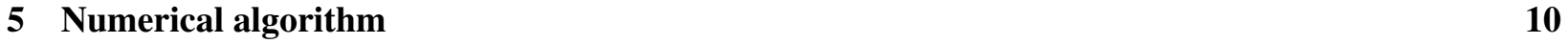

5.1 Implementation . . . . . . . . . . . . . . . . . . . . . 11

$5.1 .1 \quad$ Discretization and calculation of $\boldsymbol{E}_{j+1} \ldots \ldots \ldots \ldots \ldots \ldots \ldots$

$5.1 .2 \quad$ Local optima and saddle points . . . . . . . . . . . . . . . . . . . . 12

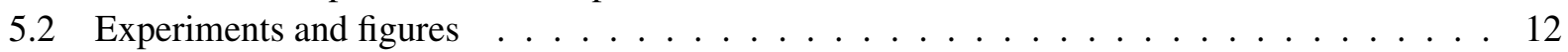

5.2 .1 Radial domain . . . . . . . . . . . . . . . . . . . . 12

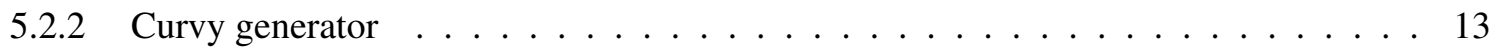

5.2 .3 Symmetry breaking . . . . . . . . . . . . . . . . . . . 13

${ }^{*}$ Published in Applied Mathematics and Computation, DOI: 10.1016/j.amc.2017.10.006. See the full citation in [EFL18].

'Department of Mathematical Sciences, The University of Nottingham Ningbo China, 199 Taikang East Road, Ningbo, Zhejiang China 315100,Behrouz.Emamizadeh@ nottingham.edu.cn

Center for Research on Embedded Systems, Halmstad University, Sweden, Amin.Farjudian@ gmail.com

${ }^{\S}$ Corresponding author

IIBeijing International Center for Mathematical Research, Peking University, China, yichen.liu07@ yahoo.com 


\section{Introduction}

Kurata and Shi [KS08] considered the problem of optimal harvesting of a marine species with the aim of minimizing harm to the species. From an applied marine economy perspective, the main result of their work is a formal argument in support of designation of no-harvesting zones.

To be more precise, consider a species of (say) fish living in a lake $\Omega$ with boundary $\partial \Omega$. The species is a source of income for fishermen, and as such, it is imperative to come up with harvesting strategies that, on the one hand, allow the fishermen to make a living, and on the other hand, minimize harm to the species and provide long term sustainability of the source of income.

In mathematical biology, reaction-diffusion equations have provided a standard model for the study of population dynamics of many species. Assuming that $w$ denotes the population density, a fairly general form of a reaction-diffusion equation may be expressed as:

$$
\partial w / \partial t=\nabla \cdot(D \nabla w)+f(w, x, t), \quad x \in \Omega, t>0,
$$

in which $f$ is regarded as the source function [Mur02, Chap. 11]. For modelling the fish population in a lake, we consider the same set of assumptions as taken by Oruganti, Shi, and Shivaji [OSS02], i. e.:

1. The fish move around via a 'random walk' in the bounded homogeneous environment $\Omega$, which implies that $D$ is constant throughout the domain. Of course, in a more general setting, it could be a function of $w, x$, and $t$.

2. The population dynamics is subject to logistic growth.

With these assumptions, one obtains the following equation:

$$
\partial w / \partial t=D \Delta w+a w(1-w / K), \quad x \in \Omega, t>0
$$

where $D>0$, and in which $a, K>0$ denote the linear reproduction rate and the carrying capacity of the environment, respectively. If the species is subject to harvesting, then a further term should be subtracted from the right hand side of (2) to account for the loss of population due to harvesting. Hence, the modified equation takes the form:

$$
\partial w / \partial t=D \Delta w+a w(1-w / K)-h(x, w), \quad x \in \Omega, t>0,
$$

in which $h(x, w)$ denotes the harvesting density per unit time.

As we are interested in qualitative properties of solutions, we would like to make some simplifications in order to turn (3) into a more manageable one. First, we assume that the harvesting density per unit time is directly proportional to the density $w$ and some harvesting effort $E(x)$ at each point $x \in \Omega$. Hence, we take $h$ to be of the form:

$$
h(x, w)=E(x) w .
$$

This is acceptable as, for instance, fishers would cast the same type of net at a point $x \in \Omega$ regardless of the density of population at that point.

Second, to avoid clutter, we would like to take the specific values that $a$ and $K$ take out of the discussion. To that end, we introduce the change of variables $w(x, t)=K u(y, t)$ and $y=\sqrt{a} x$ into 3 , which leads to:

$$
\partial u / \partial t=a(D \Delta u+u(1-u))-E(y / \sqrt{a}) u, \quad y \in \Omega^{\prime}, t>0 .
$$


Indeed, for qualitative analysis of the solutions, the parameter $a$ appearing in the right hand side has no significance in what shall follow (see, e. g., [CC89]). Hence, we normalize the value of $a$ to 1 . Combining the effects of all the simplifications, and by taking the diffusion scale $\epsilon$ to be $\epsilon:=\sqrt{D}$, we obtain:

$$
\partial u / \partial t=\epsilon^{2} \Delta u+u-u^{2}-E(y) u, \quad y \in \Omega^{\prime}, t>0 .
$$

Regarding boundary conditions, we assume that the surrounding area of the region $\Omega$ is completely inhospitable, e. g., $\Omega$ is a lake surrounded by land. This leads to the choice of Dirichlet boundary conditions. We remark here that a more general problem may be studied by considering Robin boundary conditions, but as, on the one hand, our focus in this article is not the effects of the boundary conditions, and on the other hand, with Dirichlet boundary conditions we have richer mathematical results at our disposal, we focus on the case of Dirichlet boundary conditions.

Putting together all the normalizations, simplifications, and considerations alluded to thus far leads to the dynamics of the fish population being modeled by the following reaction-diffusion equation with logistic growth:

$$
\begin{cases}\partial u / \partial t=\epsilon^{2} \Delta u+u-u^{2}-E(x) u, & x \in \Omega, t>0, \\ u(x, t)=0, & x \in \partial \Omega, t>0, \\ u(x, 0)=u_{0}(x), & x \in \Omega,\end{cases}
$$

in which:

- $\Omega \subseteq \mathbb{R}^{N}$ is a smooth domain, with $N \geq 1$;

- $u$ is the population density;

- $u_{0} \geq 0$ is an initial population density;

- $\epsilon$ is the diffusion scale;

- $E(x) \geq 0$ is the harvesting effort, and as a consequence, $E(x) u$ is the harvesting density per unit time;

- All the variables are dimensionless.

The biological energy function associated with system (4) is given as follows:

$$
\mathcal{E}_{\epsilon}(u, E):=\underbrace{\frac{\epsilon^{2}}{2} \int_{\Omega}|\nabla u|^{2} \mathrm{~d} x}_{\text {kinetic energy }} \underbrace{-\frac{1}{2} \int_{\Omega} u^{2} \mathrm{~d} x+\frac{1}{3} \int_{\Omega} u^{3} \mathrm{~d} x+\frac{1}{2} \int_{\Omega} E(x) u^{2} \mathrm{~d} x}_{\text {potential energy }},
$$

for $u \in H_{0}^{1}(\Omega)$. As time passes, this quantity decreases for $u(\cdot, t)$. To see this, observe that using the Divergence theorem, and keeping in mind that $\partial u / \partial t$ vanishes on $\partial \Omega$, one finds:

$$
\begin{aligned}
\frac{d \mathcal{E}_{\epsilon}(u(\cdot, t))}{d t} & =\int_{\Omega}\left[-\epsilon^{2} \Delta u-u+u^{2}+E(x) u\right] \frac{\partial u}{\partial t} d x \\
& =-\int_{\Omega}\left(\frac{\partial u}{\partial t}\right)^{2} d x \leq 0,
\end{aligned}
$$

for $t \in(0, \infty)$. This behavior of $\mathcal{E}_{\epsilon}(u(\cdot, t))$, coupled with the fact that $\mathcal{E}_{\epsilon}(u(x, t), E) \geq-C$, for some positive constant $C]^{1}$ guarantee that there exists a function $u_{\infty} \in H_{0}^{1}(\Omega)$ such that:

$$
\lim _{t \rightarrow \infty}\left\|u(x, t)-u_{\infty}(x)\right\|_{H_{0}^{1}(\Omega)}=0 .
$$

\footnotetext{
${ }^{1}$ See 22 on page 9
} 
The function $u_{\infty}$ satisfies the following steady state equation:

$$
\begin{cases}\epsilon^{2} \Delta u+u-u^{2}-E(x) u=0, & x \in \Omega, \\ u(x)=0, & x \in \partial \Omega .\end{cases}
$$

Of particular interest is the long term survival of the species which, according to the current formalism, is determined by the steady state solution $u$ of (6). As the effort function $E(x)$ is the only parameter under our control, we denote the solution of (6) by $u_{E}$, to stress its dependence on the harvesting strategy. Indeed, it has been shown (see, e.g., [CC89]) that when the diffusion scale $\epsilon$ is small enough, and the harvesting effort is not too aggressive-i. e., when $\{x \in \Omega \mid E(x)<1\}$ has positive measure-the population survives, in that equation (6) has a unique positive solution $u_{E}$, which satisfies: $\forall x \in \Omega: 0<u_{E}(x) \leq 1$.

Nonetheless, the survival of the species is not the same as its well-being. We have already discussed how the solution $u(x, t)$ of the parabolic equation (4) descends (in energy terms) towards a minimum energy steady state. This hints at the biological system's preference towards lower energy. By fixing $\epsilon$, and noting the dependence of $u_{E}$ on $E$ - which is the parameter under our control—we define:

$$
\Phi(E):=\frac{\epsilon^{2}}{2} \int_{\Omega}\left|\nabla u_{E}\right|^{2} \mathrm{~d} x-\frac{1}{2} \int_{\Omega} u_{E}^{2} \mathrm{~d} x+\frac{1}{3} \int_{\Omega} u_{E}^{3} \mathrm{~d} x+\frac{1}{2} \int_{\Omega} E(x) u_{E}^{2} \mathrm{~d} x .
$$

By incorporating the differential equation in (6) into the definition of $\Phi$ in (7), it can be shown that:

$$
\Phi(E)=-\frac{1}{6} \int_{\Omega} u_{E}^{3} \mathrm{~d} x
$$

To maximize the well-being of the species, our aim should be to devise a harvesting strategy $E(x)$ which minimizes $\Phi(E)$ amongst all the admissible choices for $E(x)$. This is equivalent to maximizing $\int_{\Omega} u_{E}^{3} \mathrm{~d} x$, a quantity which, at an intuitive level, may be construed as a total population distribution in the habitat. This also reinforces the idea of minimizing $\Phi$ in order to maximize the well-being of the species. To that end, Kurata and Shi [KS08] consider the following assumptions on $E(x)$ :

1. Among all the admissible choices for $E(x)$, the total effort is constant:

$$
\int_{\Omega} E(x) \mathrm{d} x=\beta \cdot|\Omega|,
$$

in which $\beta>0$ is the average effort, and $|\Omega|$ denotes the Lebesgue measure of $\Omega$.

2. The effort function $E(x)$ is non-negative and bounded, i. e., for a maximum allowable harvesting effort $M \geq \beta$ :

$$
\forall x \in \Omega: 0 \leq E(x) \leq M
$$

It is well known that the set

$$
\mathcal{E}^{\prime}:=\left\{E \in L^{\infty}(D)\left|0 \leq E(x) \leq M, \int_{\Omega} E(x) \mathrm{d} x=\beta \cdot\right| \Omega \mid\right\},
$$

which is exactly the admissible set of effort functions satisfying $(8)$ and $(9]$, is the $\sigma\left(L^{2}, L^{2}\right)$-closure of the set

$$
\mathcal{E}:=\left\{M \chi_{A} \mid A \text { is a Lebesgue-measurable subset of } \Omega \text { and }|A|=\frac{\beta}{M}|\Omega|\right\},
$$

in which $\chi_{A}$ is the characteristic function of the set $A$, see, e. g., [LE16, Lemma 4.1]. Here, $\sigma\left(L^{2}, L^{2}\right)$ denotes the weak topology on $L^{2}(\Omega)$ induced by $L^{2}(\Omega)$.

Kurata and Shi [KS08] prove that not only the energy functional $\Phi$ does attain its minimum on the admissible set $\mathcal{E}^{\prime}$, but indeed the minimum is attained on an element of $\mathcal{E}$. This, in particular, entails that the 
solution is of a 'bang-bang' type, meaning that there must be a no-harvesting zone (of maximum measure possible) set aside over which no harvesting is allowed, and outside of which harvesting is carried out with maximum allowable effort. The authors go on to prove other interesting results regarding the location and geometry of the no-harvesting zone, e.g., that it preserves the Steiner symmetry of the domain $\Omega$, and that symmetry breaking can happen over non-convex domains such as the dumbbell shaped domain and annulus. It is also argued that the no-harvesting zone must in general be away from the boundary of $\Omega$ and closer to the center of the domain.

\subsection{Our alternative approach through rearrangements of functions}

In this paper, we obtain a generalization of the result of Kurata and Shi [KS08] through the theory of rearrangements of functions as developed by G. R. Burton [Bur87, Bur89b; Bur89a]. Note that the set $\mathcal{E}$ in (10) is indeed a rearrangement class (see Definition 3 on the next page). Thus, here we attack the problem from another angle. We first consider the optimization problem over a given rearrangement class, and then prove its solvability by relaxing the admissible set to its weak closure. In contrast to the result of [KS08] where the rearrangement class had to be generated by a characteristic function, here we can consider the general multivalued generators.

The interpretation of the multivalued setting is as follows: imagine a fishing fleet consisting of boats of various capacities, and assume that, to make the deployment of each boat cost-effective, each boat is required to harvest to its maximum capacity. The problem is to find the best spatial strategy regarding the location of the boats.

Note that, if all the boats have the same capacity, the problem reduces to that considered in [KS08]. Here we solve the general multivalued problem using the theory of rearrangements of functions. Furthermore, we obtain an optimality condition, which in particular provides a generalization of the result of [KS08] regarding the location of boats. Specifically, it will be shown that the larger boats must be deployed closer to the boundary of the habitat.

Finally, we will develop an algorithm for numerical solution of the multivalued problem and present the results of some numerical experimentations. These results verify some of the theoretical conclusions of [KS08] and the current paper.

\subsection{Structure of the paper}

The rest of the paper is organized as follows:

- Section 2 contains the preliminaries on rearrangement theory and some basic properties of the steady state equation (6).

- Existence of solutions will be proven in Section 3 .

- We prove preservation of Steiner and radial symmetries in Section 4

- In Section 5, we present a numerical algorithm for finding minimizers, together with a number of figures from our experiments that verify the theory.

- To avoid a large gap in the flow of the content, we have included the lengthy proof of Lemma 13 from Section 3-in Section 6. This is an essential lemma which deals with the fundamental properties of the energy functional $\Phi$.

- In Section7, we finish the paper with some concluding remarks regarding where the current result fits in the general area of applications of rearrangement optimization problems. 


\section{Preliminaries}

The material in this section includes some basic results from the theory of rearrangements of functions, and some background on the steady state equation (6), which we will need later on.

\subsection{Rearrangements of functions}

For a Lebesgue measurable function $h: \Omega \rightarrow[0, \infty)$ and $\alpha \geq 0$, we let:

$$
\lambda_{h}(\alpha):=|\{x \in \Omega \mid h(x) \geq \alpha\}| .
$$

Definition 1. Let $g_{0}: \Omega_{1} \rightarrow[0, \infty)$ and $g: \Omega_{2} \rightarrow[0, \infty)$ be Lebesgue measurable. We say that $g$ is a rearrangement of $g_{0}$ if and only if $\forall \alpha \geq 0: \lambda_{g_{0}}(\alpha)=\lambda_{g}(\alpha)$.

Definition 2. For a Lebesgue measurable $g: \Omega \rightarrow[0, \infty)$, the essentially unique decreasing rearrangement $g^{\Delta}$ of $g$ is defined on $(0,|\Omega|)$ by $g^{\Delta}(s):=\max \left\{\alpha \in \mathbb{R} \mid \lambda_{g}(\alpha) \geq s\right\}$. The essentially unique increasing rearrangement $g_{\Delta}$ of $g$ is defined by $g_{\Delta}(s):=g^{\Delta}(|\Omega|-s)$.

Definition 3. The rearrangement class $\mathcal{R}\left(g_{0}\right)$ generated by $g_{0}$ is defined as follows:

$$
\mathcal{R}\left(g_{0}\right):=\left\{g: \Omega \rightarrow[0, \infty) \mid g \text { is a rearrangement of } g_{0}\right\} .
$$

When the generator is clear from the context, we may simply write $\mathcal{R}$.

Definition 4. For a function $f: \Omega \rightarrow \mathbb{R}$, we say that the graph of $f$ has no significant flat zones on $\Omega$ if $\forall c \in \mathbb{R}:|\{x \in \Omega \mid f(x)=c\}|=0$.

Lemma 5. Let $g_{0} \in L^{\infty}(\Omega)^{+}$, where $L^{\infty}(\Omega)^{+}$denotes the positive cone generated by non-negative functions in $L^{\infty}(\Omega)$. Let $\overline{\mathcal{R}}$ be the weak closure of $\mathcal{R}:=\mathcal{R}\left(g_{0}\right)$ in $L^{2}(\Omega)$. Then, $\overline{\mathcal{R}} \subseteq L^{\infty}(\Omega)$, and $\forall g \in \overline{\mathcal{R}}:\|g\|_{\infty} \leq\left\|g_{0}\right\|_{\infty}$.

Proof. Let $g \in \overline{\mathcal{R}}$, and assume that $D:=\left\{x \in \Omega \mid g(x)>\left\|g_{0}\right\|_{\infty}\right\}$ has a positive Lebesgue measure. As $g \in \overline{\mathcal{R}}$, there exists $\left\{g_{n}\right\} \subseteq \mathcal{R}$ such that $g_{n} \rightarrow g$ in $L^{2}(\Omega)$. Then, we have:

$$
\int_{D} g_{n} \mathrm{~d} x=\int_{\Omega} g_{n} \chi_{D} \mathrm{~d} x \rightarrow \int_{\Omega} g \chi_{D} \mathrm{~d} x=\int_{D} g \mathrm{~d} x .
$$

As each $g_{n}$ is in the rearrangement class of $g_{0}$, we have $\int_{D} g_{n} \mathrm{~d} x \leq\left\|g_{0}\right\|_{\infty}|D|$. On the other hand, from the definition of $D$, in conjunction with $(11)$, we deduce:

$$
\left\|g_{0}\right\|_{\infty}|D|<\int_{D} g \mathrm{~d} x=\lim _{n \rightarrow \infty} \int_{D} g_{n} \mathrm{~d} x \leq\left\|g_{0}\right\|_{\infty}|D|,
$$

which is a contradiction. As a result, the measure of $D$ is zero, and $\|g\|_{\infty} \leq\left\|g_{0}\right\|_{\infty}$.

Lemma 6. Assume that $\left\{g_{n}\right\}_{n \in \mathbb{N}}$ is a sequence of non-negative functions in $L^{\infty}(\Omega), g \in L^{2}(\Omega)$, and $g_{n} \rightarrow g$ in $L^{2}(\Omega)$. Then, $g$ is non-negative a.e. in $\Omega$.

Proof. By Mazur's Lemma, there exists a sequence $\left\{v_{n}\right\}$ in the convex hull of the set $\left\{g_{n} \mid n \in \mathbb{N}\right\}$, such that $v_{n} \rightarrow g$ in $L^{2}(\Omega)$. Therefore, $v_{n} \rightarrow g$ in measure. Whence, there exists a subsequence of $\left\{v_{n}\right\}$ which converges to $g$ almost everywhere in $\Omega$. This completes the proof.

Lemma 7. Let $g_{0} \in L^{2}(\Omega)^{+}$, and let $\mathcal{R}:=\mathcal{R}\left(g_{0}\right)$. Suppose $\Psi: \overline{\mathcal{R}} \rightarrow \mathbb{R}$ is convex, weakly sequentially continuous, and satisfies the following:

$$
\lim _{t \rightarrow 0^{+}} \frac{\Psi(u+t(v-u))-\Psi(u)}{t}=\int_{\Omega} G(u)(v-u) \mathrm{d} x, \quad \forall v, u \in \overline{\mathcal{R}},
$$

where $G: \overline{\mathcal{R}} \rightarrow L^{2}(D)$ is an operator. Then, we have: 
(i) $\Psi$ attains a maximum value relative to $\mathcal{R}$.

(ii) Let $\hat{u}$ be a maximizer for $\Psi$ relative to $\mathcal{R}$, and suppose $\Psi$ is locally strictly convex at $\hat{u}$, i.e.

$$
\exists \epsilon>0: \Psi \text { is strictly convex on } \overline{\mathcal{R}} \cap B(\hat{u}, \epsilon),
$$

Then, $\hat{u}=\eta \circ G(\hat{u})$ a.e. in $\Omega$, for some non-decreasing function $\eta$.

\section{Proof.}

(i) We begin by considering the following maximization problem:

$$
\sup _{u \in \overline{\mathcal{R}}} \Psi(u) .
$$

Since $\Psi$ is weakly continuous, and $\overline{\mathcal{R}}$ is weakly compact, 13 is solvable. Let $\bar{u} \in \overline{\mathcal{R}}$ be a solution. By applying the formula (12), we infer that $\bar{u}$ maximizes the continuous linear functional $\mathcal{L}(h):=$ $\int_{\Omega} G(\bar{u}) h \mathrm{~d} x$ relative to $h \in \overline{\mathcal{R}}$. It is known that the functional $\mathcal{L}$ has a maximizer $\tilde{u}$ relative to $\mathcal{R}[$ Bur87, Theorem 4]. Since $\mathcal{L}$ is weakly continuous, it follows that $\tilde{u}$ maximizes $\mathcal{L}$ relative to $\overline{\mathcal{R}}$ as well. Whence, in particular, $\mathcal{L}(\bar{u}) \leq \mathcal{L}(\tilde{u})$. By using the convexity of $\Psi$ and the formula $(12)$, we have:

$$
\Psi(\tilde{u})-\Psi(\bar{u}) \geq \lim _{t \rightarrow 0^{+}} \frac{\Psi(\bar{u}+t(\tilde{u}-\bar{u}))-\Psi(\bar{u})}{t}=\int_{\Omega} G(\bar{u})(\tilde{u}-\bar{u}) \mathrm{d} x=\mathcal{L}(\tilde{u}-\bar{u}) \geq 0 .
$$

Thus, $\Psi(\tilde{u})=\Psi(\bar{u})$, and $\tilde{u} \in \mathcal{R}$ is a solution of $(13)$, as desired.

(ii) Let us fix a maximizer $\hat{u}$ satisfying the local strict convexity. For any $v \in \mathcal{R}$, there exists $t>0$ small enough such that:

$$
0 \geq \Psi(v)-\Psi(\hat{u})>\frac{\Psi(\hat{u}+t(v-\hat{u}))-\Psi(\hat{u})}{t} \geq \int_{\Omega} G(\hat{u})(v-\hat{u}) \mathrm{d} x,
$$

where the second inequality follows from local strict convexity of $\Psi$ at $\hat{u}$, and the third inequality is derived from the convexity of $\Psi$. Thus, $\hat{u}$ is the unique maximizer of the continuous linear functional $\mathcal{L}_{1}(v):=\int_{\Omega} G(\hat{u}) v \mathrm{~d} x$ relative to $v \in \mathcal{R}$. By applying Theorem 5 in [Bur87], we infer $\hat{u}=\eta \circ G(\hat{u})$ a.e. in $\Omega$, for some non-decreasing function $\eta$, as desired.

\subsection{Steady state equation (6)}

We need the following result regarding existence and uniqueness of solutions of the boundary value problem (BVP) (6):

Proposition 8. Assume that $|\{x \in \Omega \mid E(x)<1\}|>0$. Then, there exists an $\epsilon_{1}=\epsilon_{1}(\Omega, E)>0$ such that:

- When $\epsilon \geq \epsilon_{1}$, the BVP (6) has only the trivial solution $u_{E}=0$.

- When $0<\epsilon<\epsilon_{1}$, the BVP (6) has a unique positive solution, which is positive throughout the (interior of the) domain, and bounded above by 1, i. e.:

$$
\forall x \in \Omega: 0<u_{E}(x) \leq 1 .
$$

Furthermore, this solution is globally asymptotically stable, in the sense that, for any $u_{0} \in L^{2}(\Omega)$ with $u_{0}(x) \geq 0$ for all $x \in \Omega$, the solution $u(x, t)$ of the parabolic equation (4) satisfies:

$$
\lim _{t \rightarrow \infty}\left\|u(x, t)-u_{E}(x)\right\|_{H_{0}^{1}(\Omega)}=0 .
$$


Proof. See [CC89, Sect. 2].

Remark 9. There is indeed an explicit description of the value $\epsilon_{1}$ of Proposition 8 Consider the following eigenvalue problem:

$$
\begin{cases}-\Delta z=\lambda(1-E(x)) z, & x \in \Omega, \\ z=0, & x \in \partial \Omega,\end{cases}
$$

and let $\lambda_{1}^{+}$be the smallest positive eigenvalue of $\sqrt{14}$. Then $\epsilon_{1}=\frac{1}{\sqrt{\lambda_{1}^{+}}}$. For further details, see [CC89. Sect. 2].

\section{Existence of solutions}

Let us first go through the formal statement of the problem. We take a non-negative function $E_{0} \in L^{\infty}(\Omega)$ as the generator of the rearrangement class $\mathcal{R}\left(E_{0}\right)$, where $E_{0}$ satisfies:

$$
\frac{1}{|\Omega|} \int_{\Omega} E_{0}(x) \mathrm{d} x<1 .
$$

Each element of this rearrangement class represents a harvesting strategy, and for each $E \in \mathcal{R}\left(E_{0}\right)$ and $x \in \Omega$, the value $E(x)$ corresponds to the harvesting capacity of the boat located at $x$. The special case where $E_{0}$ is a characteristic function corresponds to the one considered in [KS08], where all the boats have the same capacity.

Since $\epsilon_{1}$ depends on $E$ when $\Omega$ is fixed, we need to maximize the $\epsilon_{1}$ among the rearrangement class $\mathcal{R}\left(E_{0}\right)$, i. e.:

$$
\epsilon_{2}\left(E_{0}\right):=\sup _{E \in \mathcal{R}\left(E_{0}\right)} \epsilon_{1}(\Omega, E) .
$$

Proposition 10. Let $E_{0}$ be a non-negative function in $L^{\infty}(\Omega)$ which satisfies $(15)$, and let $\mathcal{R}:=\mathcal{R}\left(E_{0}\right)$. Then, the maximization problem $(16)$ is solvable. Furthermore, for any maximizer $\tilde{E}$, there exists a non-decreasing function $\varphi$ such that $\tilde{E}=\varphi \circ u_{\tilde{E}}$.

Proof. By Remark 9, the maximization problem (16) is equivalent to the following minimization problem:

$$
\frac{1}{\epsilon_{2}\left(E_{0}\right)^{2}}=\inf _{E \in \mathcal{R}\left(E_{0}\right)} \lambda_{1}^{+}(E)
$$

By applying Theorem 1 (i) in [CCP13], we have the desired results.

Remark 11. We know that $\epsilon_{1}(\Omega, \cdot)$ is continuous on $\overline{\mathcal{R}}\left[\right.$ [CCP13. Prop. 1 (i)]. For $\epsilon<\epsilon_{2}$, there exists $E_{1} \in \mathcal{R}\left(E_{0}\right)$ such that $\epsilon<\epsilon_{1}\left(\Omega, E_{1}\right)$, while it is possible to have $\epsilon \geq \epsilon_{1}\left(\Omega, E_{2}\right)$ for some other $E_{2} \in \mathcal{R}\left(E_{0}\right)$.

Now, let us fix a positive $\epsilon<\epsilon_{2}\left(E_{0}\right)$. We are interested in minimizing harm to the species, which corresponds to minimization of the functional:

$$
\Phi(E):=\frac{\epsilon^{2}}{2} \int_{\Omega}\left|\nabla u_{E}\right|^{2} \mathrm{~d} x-\frac{1}{2} \int_{\Omega} u_{E}^{2} \mathrm{~d} x+\frac{1}{3} \int_{\Omega} u_{E}^{3} \mathrm{~d} x+\frac{1}{2} \int_{\Omega} E(x) u_{E}^{2} \mathrm{~d} x=-\frac{1}{6} \int_{\Omega} u_{E}^{3} \mathrm{~d} x
$$

over the set $\mathcal{R}\left(E_{0}\right)$ of all the admissible strategies. We denote this problem by the usual notation:

$$
\inf _{E \in \mathcal{R}\left(E_{0}\right)} \Phi(E) .
$$

To prove the existence of solutions for (19), we need to explore some properties of the energy functional $\Phi$. 
Lemma 12. If - by a slight abuse of notation-we define:

$$
\Phi(v, E):=\frac{\epsilon^{2}}{2} \int_{\Omega}|\nabla v|^{2} \mathrm{~d} x-\frac{1}{2} \int_{\Omega} v^{2} \mathrm{~d} x+\frac{1}{3} \int_{\Omega} v^{3} \mathrm{~d} x+\frac{1}{2} \int_{\Omega} E(x) v^{2} \mathrm{~d} x,
$$

then we have:

$$
\Phi(E)=\inf _{v \in H_{0}^{1}(\Omega)^{+}} \Phi(v, E) .
$$

Indeed, for each fixed $E$, the solution $u_{E}$ of the $B V P(6)$ is the unique minimizer of $\Phi(v, E)$ with respect to $v \in H_{0}^{1}(\Omega)^{+}$.

Proof. Since $E(x) \geq 0$, for every $v \in H_{0}^{1}(\Omega)^{+}$, we have

$$
\begin{aligned}
\Phi(v, E) & \geq \frac{\epsilon^{2}}{2}\|v\|_{H_{0}^{1}(\Omega)}^{2}-\frac{1}{2} \int_{\Omega} v^{2} \mathrm{~d} x+\frac{1}{3} \int_{\Omega} v^{3} \mathrm{~d} x=\frac{\epsilon^{2}}{2}\|v\|_{H_{0}^{1}(\Omega)}^{2}+\frac{1}{6} \int_{\Omega} v^{2}(2 v-3) \mathrm{d} x \\
& \geq \frac{\epsilon^{2}}{2}\|v\|_{H_{0}^{1}(\Omega)}^{2}+\frac{1}{6} \int_{\left\{v<\frac{3}{2}\right\}} v^{2}(2 v-3) \mathrm{d} x \geq \frac{\epsilon^{2}}{2}\|v\|_{H_{0}^{1}(\Omega)}^{2}-\frac{9}{8}|\Omega| .
\end{aligned}
$$

So, $\Phi(\cdot, E)$ is coercive and bounded below on $H_{0}^{1}(\Omega)^{+}$. By applying the direct method of calculus of variations, we infer the existence of a minimizer $v$ for (21). We intend to show that $v=u_{E}$, in the sense of Proposition 8. To that end, we apply the classical techniques of variational inequalities, (see, e. g., [Eva10, Sect. 8.4.2]). It follows that:

$$
\left\{\begin{aligned}
v \geq 0,-\epsilon^{2} \Delta v & \geq(1-E(x)) v-v^{2}, & & \text { in } \Omega, \\
-\epsilon^{2} \Delta v & =(1-E(x)) v-v^{2}, & & \text { on } \Omega \cap\{v>0\} .
\end{aligned}\right.
$$

However, in the region $\{x \in \Omega \mid v(x)=0\}$, the differential equation in the second line of $(23)$ is trivially satisfied. This implies that the minimizer satisfies $v=0$ or $v=u_{E}$. If $\epsilon \geq \epsilon(\Omega, E)$, we must have $v=0$. On the other hand, if $\epsilon<\epsilon(\Omega, E)$, we infer that $v=u_{E}$ is the unique positive solution, since $\Phi\left(u_{E}, E\right)=$ $-\frac{1}{6} \int_{\Omega} u_{E}^{3} \mathrm{~d} x<0$.

Lemma 13. Let $E_{0}$ be a non-negative function in $L^{\infty}(\Omega)$ which satisfies $(15)$, and let $\mathcal{R}:=\mathcal{R}\left(E_{0}\right)$. Suppose that $0<\epsilon<\epsilon_{2}\left(E_{0}\right)$. The functional $\Phi: \overline{\mathcal{R}} \rightarrow \mathbb{R}$ satisfies the following properties:

(i) $\Phi$ is weakly sequentially continuous w.r.t. the $L^{2}$ topology.

(ii) $\Phi$ is concave. Moreover, for any $E \in \overline{\mathcal{R}}$ with $u_{E}>0$, $\Phi$ is locally strictly concave at $E$, i. e.:

$$
\exists \epsilon>0: \Phi \text { is strictly concave on } \overline{\mathcal{R}} \cap B(E, \epsilon) .
$$

(iii) For any given $E$ and $F$ in $\overline{\mathcal{R}}$, by assigning $v:=F-E$, we get:

$$
\lim _{t \rightarrow 0^{+}} \frac{\Phi(E+t v)-\Phi(E)}{t}=\frac{1}{2} \int_{\Omega} v u_{E}^{2} \mathrm{~d} x
$$

Proof. See Section6.

Based on the properties of the energy functional as stated in Lemma 13, we can apply the theory of Burton [Bur87] to obtain the following existence result:

Theorem 1. Let $E_{0}$ be a non-negative function in $L^{\infty}(\Omega)$ which satisfies $(15)$, and let $0<\epsilon<\epsilon_{2}\left(E_{0}\right)$. Then, the minimization problem (19) is solvable. Furthermore, for any minimizer $E^{*}$, there exists a non-increasing function $\check{\phi}$ such that:

$$
E^{*}=\check{\phi} \circ u_{E^{*}}
$$


Proof. Define $\Psi(E):=-\Phi(E)$. By Lemma 13 , the functional $\Psi$ is weakly sequentially continuous w.r.t. the $L^{2}$ topology, convex, and satisfies the formula $\sqrt{12}$ with $G(E)=-\frac{1}{2} u_{E}^{2}$. Thus, we can apply Lemma 7 to deduce that the maximization problem:

$$
\sup _{E \in \mathcal{R}\left(E_{0}\right)} \Psi(E)
$$

is solvable.

Let us fix a maximizer $E^{*} \in \mathcal{R}\left(E_{0}\right)$, and note that we must have $u_{E^{*}}>0$ by Remark 11. It follows from Lemma 13 (ii) that $\Psi$ is locally strictly convex at $E^{*}$. By Lemma 7 (ii), we have $E^{*}=\hat{\phi} \circ\left(-\frac{1}{2} u_{E^{*}}^{2}\right)$, for some non-decreasing function $\hat{\phi}$. This implies that $E^{*}=\breve{\phi} \circ u_{E^{*}}$, in which $\check{\phi}:[0, \infty) \rightarrow \mathbb{R}$, defined by $\breve{\phi}(t):=\hat{\phi}\left(-t^{2} / 2\right)$, is non-increasing.

It should be obvious that any solution of the maximization problem 26 is a solution of our main minimization problem (19), and vice versa. Hence, the proof is complete.

\section{Symmetry results}

In this section, we investigate preservation of Steiner and radial symmetries by the minimizer, when $\Omega$ has the respective symmetries.

Theorem 2. Suppose that $\left|\left\{x \in \Omega \mid E_{0}(x)=\left\|E_{0}\right\|_{\infty}\right\}\right|>0$. If $\Omega$ is Steiner symmetric with respect to a hyperplane $P$, then $E^{*}$ is increasingly Steiner symmetric with respect to the hyperplane $P$.

Proof. The proof is similar to Theorem 2.5 in [KS08]. Without loss of generality, we assume that $\Omega$ is Steiner symmetric with respect to the hyperplane $x_{1}=0$. Let $E^{*}$ be a minimizer of the optimization problem with $E^{*}=\breve{\phi} \circ u_{E^{*}}$ for a non-increasing $\breve{\phi}$ as shown in Theorem 1 . Then, we have:

$$
-\epsilon^{2} \Delta u_{E^{*}}=\left(1-\left\|E_{0}\right\|_{\infty}-u_{E^{*}}\right) u_{E^{*}}+\left(\left\|E_{0}\right\|_{\infty}-\breve{\phi} \circ u_{E^{*}}\right) u_{E^{*}} .
$$

Let us set $f_{1}(u):=\left(1-\left\|E_{0}\right\|_{\infty}-u\right) u$ and $f_{2}(u):=\left(\left\|E_{0}\right\|_{\infty}-\breve{\phi} \circ u\right) u$. By applying Theorem 3.6 in [Fra00], we conclude that $u_{E^{*}}$ is decreasingly Steiner symmetric with respect to the hyperplane $x_{1}=0$. Recalling that $E^{*}=\breve{\phi} \circ u_{E^{*}}$, we infer that $E^{*}$ is increasingly Steiner symmetric with respect to the hyperplane $x_{1}=0$.

The following is a direct consequence:

Corollary 14. Suppose that $\left|\left\{x \in \Omega \mid E_{0}(x)=\left\|E_{0}\right\|_{\infty}\right\}\right|>0$. If $\Omega$ is a ball centered at the origin, then $E^{*}$ is increasingly Schwarz symmetric with respect to the origin. Moreover, $E^{*}$ is the unique minimizer of the optimization problem (19).

Remark 15. The assumption $\left|\left\{x \in \Omega \mid E_{0}(x)=\left\|E_{0}\right\|_{\infty}\right\}\right|>0$ is a reasonable one, as in practice we expect the maximal harvesting to be taken over a region rather than at a point.

\section{Numerical algorithm}

The gradient formula (24) of Lemma 13, together with the optimality condition 25 of Theorem 1 , give us the necessary machinery for devising a numerical algorithm for solving the minimization problem (19), which is only practical when the generator $E_{0}$ is a characteristic function. For an example of such an algorithm, see [EFZR16].

Fortunately, for the current problem we can devise an algorithm based on the approach of Eydeland and Turkington [ET88], which was also applied to a rearrangement problem by Elcrat and Nicolio [EN95]. The pseudocode in Fig. 1 is a simplified account of the main iteration in our algorithm.

Proposition 16. The iteration of Fig. 1 1 on the following page generates a minimizing sequence, i. e., at every iteration $j$ we have:

$$
\Phi\left(E_{j+1}\right) \leq \Phi\left(E_{j}\right)
$$


Figure 1 Iteration for generating a minimizing sequence.

Input: A generator $E_{0}$, and an $\epsilon>0$.

Output: An approximation of a local minimum.

1. Start with $E_{0}$;

2. Set $j:=0$;

3. Repeat

4. $\quad E_{j+1}:=\arg \min _{E \in \mathcal{R}\left(E_{0}\right)} \int_{\Omega} E u_{j} \mathrm{~d} x$, where $u_{j}:=u_{E_{j}} ;$

5. $\quad j:=j+1$

6. Until $\frac{\left\|E_{j+1}-E_{j}\right\|_{\infty}}{\left\|E_{0}\right\|_{\infty}}<\epsilon$.

Proof. This is straightforward, as we have:

$$
\begin{aligned}
\Phi\left(E_{j}\right)+\frac{1}{2} \int_{\Omega}\left(E_{j+1}-E_{j}\right) u_{j}^{2} \mathrm{~d} x & =\Phi\left(u_{j}, E_{j+1}\right) \\
(\text { by Lemma 12) } & \geq \Phi\left(u_{j+1}, E_{j+1}\right) \\
& =\Phi\left(E_{j+1}\right) .
\end{aligned}
$$

Note that by line 4 of the iteration, we have chosen $E_{j+1}$ to minimize the functional $L_{1}(h):=\int_{\Omega} h u_{j} \mathrm{~d} x$ relative to the rearrangement class $\mathcal{R}\left(E_{0}\right)$. But as $u_{j}$ is non-negative, then $E_{j+1}$ also minimizes $L_{2}(h):=$ $\int_{\Omega} h u_{j}^{2} \mathrm{~d} x$. This implies that:

$$
\frac{1}{2} \int_{\Omega}\left(E_{j+1}-E_{j}\right) u_{j}^{2} \mathrm{~d} x \leq 0,
$$

which, together with 28, prove inequality 27).

\subsection{Implementation}

We have implemented an algorithm for solving (19) in dimension $N=2$, using the iteration of Fig. 1. which provides the basic machinery for generating a minimizing sequence. Here, we discuss a couple of issues regarding concrete implementation of the algorithm.

\subsubsection{Discretization and calculation of $E_{j+1}$}

The only non-trivial step of the iteration is the calculation of $E_{j+1}$ in Line 4. Suppose that we have a mesh $\mathcal{D}=\left\{D_{i} \mid 0 \leq i \leq P-1\right\}$ (for some $P \in \mathbb{N}$ ) over the domain $\Omega \subseteq \mathbb{R}^{2}$, such that $\Omega=\cup \mathcal{D}$. The corresponding discretization of the generator provides a list of $P$ values, which we sort in ascending order to obtain:

$$
E_{0}^{(0)} \leq E_{0}^{(1)} \leq \ldots \leq E_{0}^{(P-1)} .
$$

These are the various values that the (discretized) generator takes over (say, the centroid of) each finite element $D_{i}$.

At each iteration $j$, we sort the values of $u_{j}$ over the (say, centroids of the) finite elements, in descending order, to obtain:

$$
u_{j}\left(D_{\sigma(0)}\right) \geq u_{j}\left(D_{\sigma(1)}\right) \geq \ldots \geq u_{j}\left(D_{\sigma(P-1)}\right)
$$

in which $\sigma:\{0,1, \ldots, P-1\} \rightarrow\{0,1, \ldots, P-1\}$ is a permutation. To obtain $E_{j+1}$, it suffices to assign the value $E_{0}^{(k)}$ to the finite element $D_{\sigma(k)}$, for $k \in\{0,1, \ldots, P-1\}$.

Thus, assuming that $u_{j}$ has already been obtained, the calculation of $E_{j+1}$ requires a simple sorting of $P$ elements. 
Remark 17. Care must be taken as the current procedure relies on $u_{j}$ having no significant flat zones. Indeed, by assuming:

$$
\forall c \in[0,1):|\{x \in \Omega \mid E(x)=c\}|=0,
$$

it can be shown that $u_{E}$ does not have any significant flat zones. We do not have a proof for the general case, but in our numerical simulations, none of the $u_{j}$ 's have any flat zones, so we can apply the above procedure.

\subsubsection{Local optima and saddle points}

We have neither proven nor disproven the existence of non-global local optima or saddle points. Nonetheless, our numerical experiments strongly indicate the existence of local optima, from which the algorithm cannot escape except through the use of randomization. The local optima show themselves more clearly over the dumbbell shaped domain, in the presence of a very small no-harvesting zone (or zones with very low harvesting effort).

To escape local optima (and potential saddle points), we have enhances the algorithm with randomization through Simulated Annealing. Of course, with randomized algorithms the best one may get is a near-optimal solution, and due to the huge size of the search space, a brute force search is impractical. For more details, please see [EFZR16].

\subsection{Experiments and figures}

We present the results of some of our numerical experiments. All of the figures have been produced in MATLAB $^{\circledR}$. The computations in each case have taken less than 30 seconds on a personal laptop.

\subsubsection{Radial domain}

We have considered a disc-shaped lake of area 1 . We want to cover $35 \%$ of the domain with larger boats, $45 \%$ with medium-sized boats, and leave the remaining $20 \%$ as the no-harvesting zone. Figure 2 below shows the result of running our algorithm on this domain.

Note that the generator of the rearrangement class is 3-valued. The optimal strategy, as shown in the middle of the figure, suggests that the smaller boats should be positioned closer to the no-harvesting zone, which is in the middle. The larger boats harvest around the boundary. Of course, this is intuitively expected. Note also that radial symmetry is preserved.

Figure 2 From left to right: the decreasing rearrangement $E_{0}^{\Delta}$ of the 3-valued generator $E_{0}$, the best harvesting strategy $E^{*}$, and the population density $u_{E^{*}}$ under this optimal strategy. The no-harvesting zone is the blue zone in the middle, the green zone is where the medium sized boats should be sent, and the red strip around the border is where the largest boats should do their harvesting.

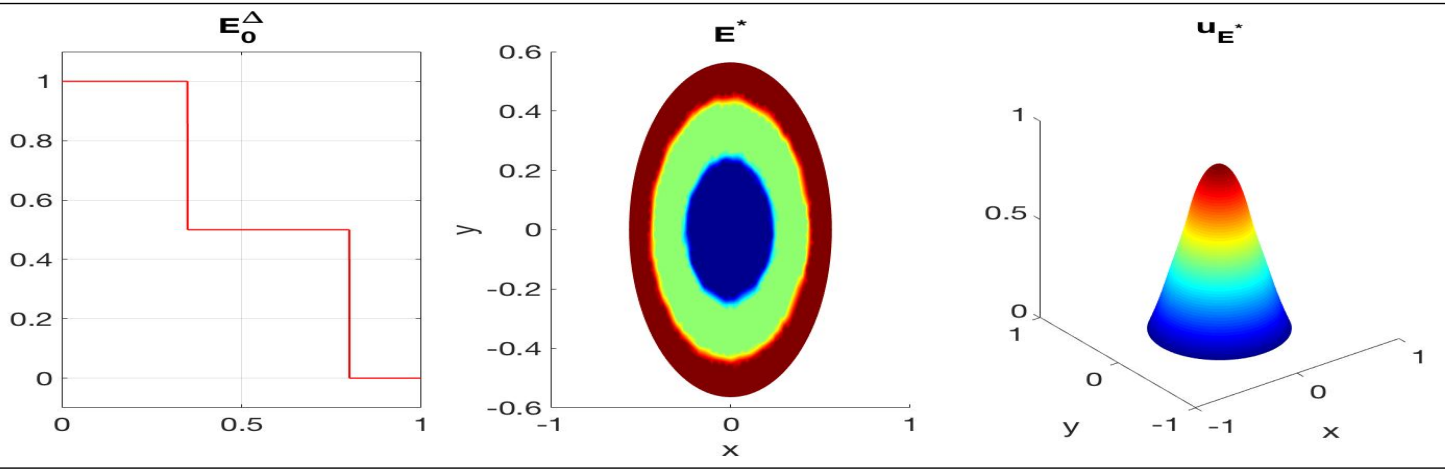




\subsubsection{Curvy generator}

To show that our algorithm works for general multi-valued generators, we have considered a generator with no flat zones at all, over a cardioid of area 20. The result is shown in Fig. 3 below. Starting from a random initial guess $E_{0}$, the algorithm reaches a stable result in about 60 iterations, under 20 seconds, and with relative error less than $10^{-2.8}$. Here, the relative error at each iteration $j$ is calculated by:

$$
\text { relative error at iteration } j=\frac{\left\|E_{j+1}-E_{j}\right\|_{\infty}}{\left\|E_{0}\right\|_{\infty}}=\left\|E_{j+1}-E_{j}\right\|_{\infty}
$$

as $\left\|E_{0}\right\|_{\infty}=1$. A further 100 iterations takes the relative error to just below $10^{-3}$, but makes no noticeable dent in the optimal energy.

Figure 3 Multivalued generator on the cardioid: Again note that the harvesting should be less aggressive towards the center.
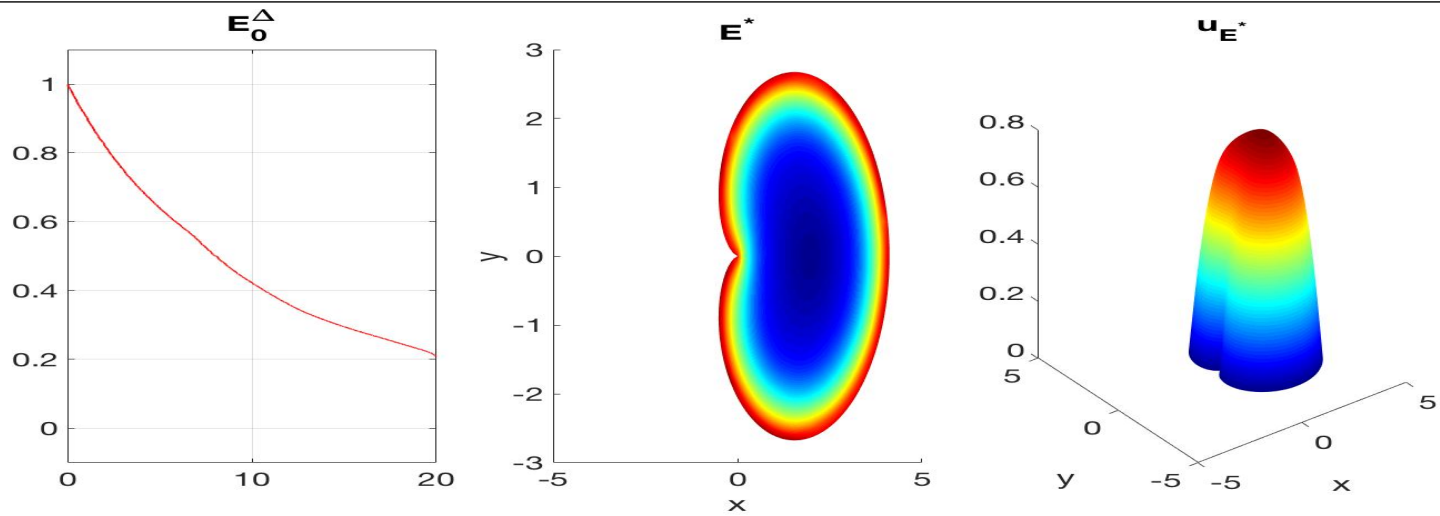

\subsubsection{Symmetry breaking}

Kurata and Shi [KS08] prove that symmetry breaking can occur for the 2-valued case in dumbbell-shaped and annulus-shaped domains, when the no-harvesting zone is quite small.

In the case of the dumbbell-shaped domain (Fig. 4 on the next page) the two patches must be relatively large, and the channel between them should be relatively narrow. We have considered a domain of area 1 , with the no-harvesting zone taking up $8 \%$ of the area.

In the case of the annulus, the radius of the two inner and outer circles should be relatively large, and the strip should be quite narrow (Fig. 5 on the following page). Here we have taken the domain to have area 10 , and the no-harvesting zone to take up $5 \%$ of the total area of the lake.

In both cases, our experiments produce symmetry breaking, as predicted by the theory. Nonetheless, in the case of the dumbbell-shaped domain, Steiner symmetry is preserved, as proven by Kurata and Shi [KS08], and also generalized in Theorem 2 of the current document.

\section{Proof of Lemma 13}

(i) We follow the approach taken in proving Lemma 3.3 (i) in [EL15]. Consider a sequence $\left\{E_{n} \mid n \in \mathbb{N}\right\} \subseteq$ $\overline{\mathcal{R}}$ and a function $\hat{E} \in \overline{\mathcal{R}}$, such that $E_{n} \rightarrow \hat{E}$ in $L^{2}(\Omega)$. For simplicity, let us set $u_{n}:=u_{E_{n}}$ and $u:=u_{\hat{E}}$. By applying Lemma 12 , we infer: 
Figure 4 Verifying symmetry breaking in the Dumbbell shaped domain: Note that the no-harvesting zone is the blue zone in the right patch.

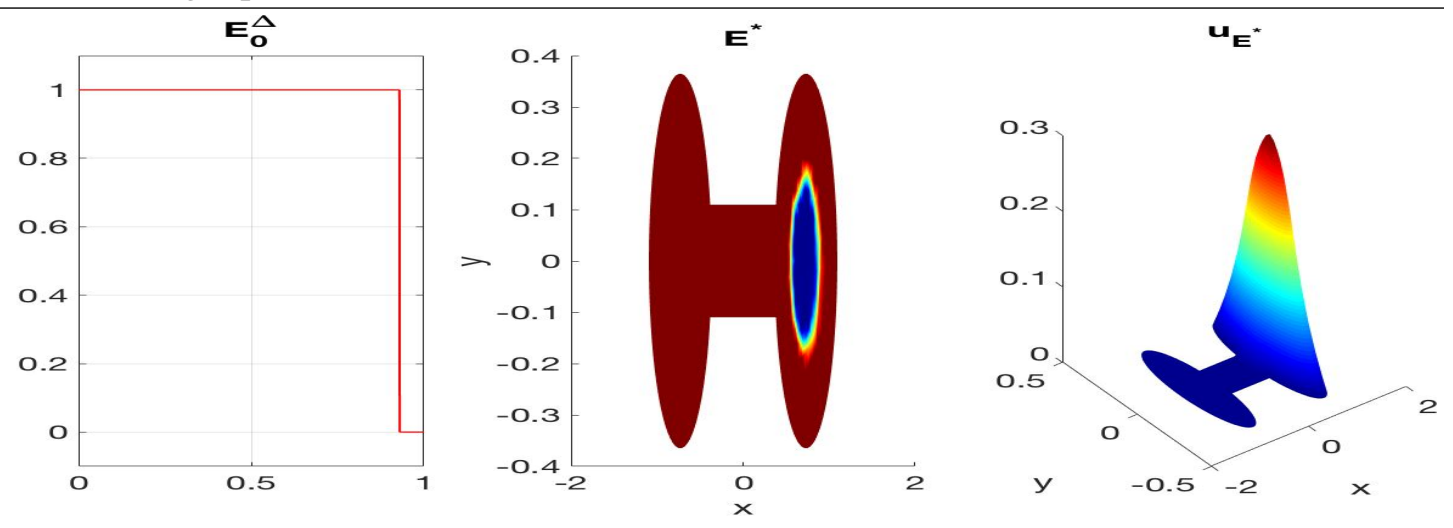

Figure 5 Verifying symmetry breaking in Annulus shaped domain: The no-harvesting zone comprises the two small blue areas.

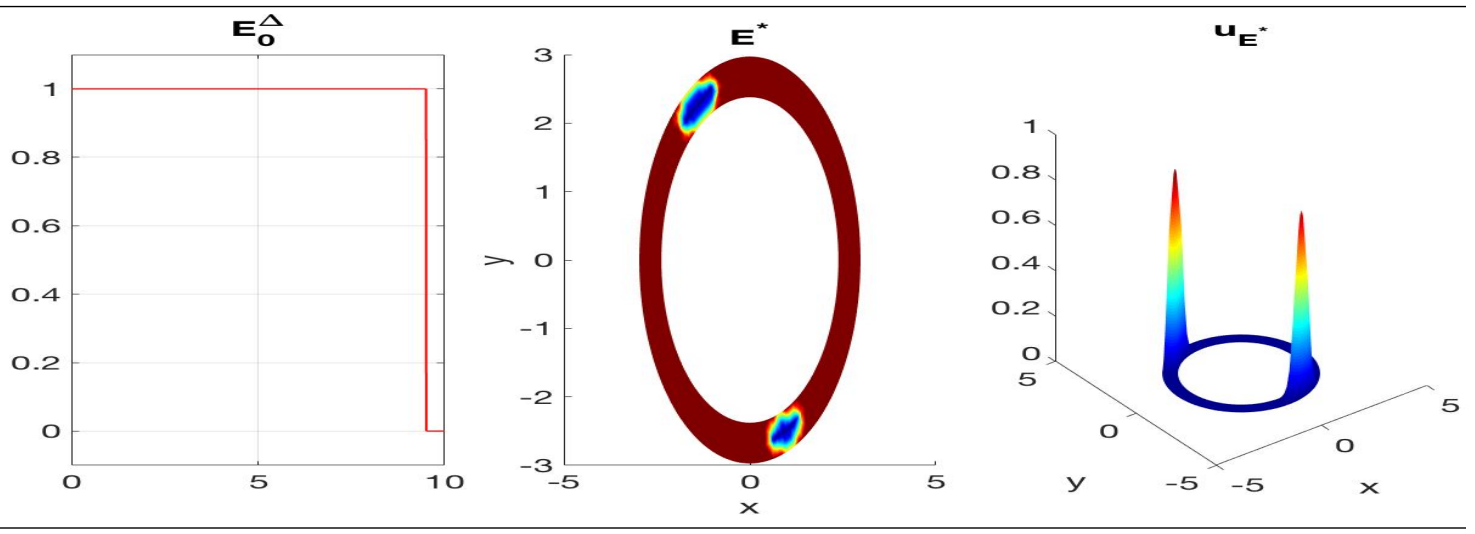

$$
\begin{aligned}
\Phi\left(E_{n}\right)+\frac{1}{2} \int_{\Omega}\left(\hat{E}-E_{n}\right) u_{n}^{2} \mathrm{~d} x & =\Phi\left(u_{n}, E_{n}\right)+\frac{1}{2} \int_{\Omega}\left(\hat{E}-E_{n}\right) u_{n}^{2} \mathrm{~d} x=\Phi\left(u_{n}, \hat{E}\right) \\
& \geq \Phi(\hat{E})=\Phi\left(u, E_{n}\right)+\frac{1}{2} \int_{\Omega}\left(\hat{E}-E_{n}\right) u^{2} \mathrm{~d} x \\
& \geq \Phi\left(u_{n}, E_{n}\right)+\frac{1}{2} \int_{\Omega}\left(\hat{E}-E_{n}\right) u^{2} \mathrm{~d} x=\Phi\left(E_{n}\right)+\frac{1}{2} \int_{\Omega}\left(\hat{E}-E_{n}\right) u^{2} \mathrm{~d} x .
\end{aligned}
$$

So, it suffices to show that:

$$
\lim _{n \rightarrow \infty} \int_{\Omega}\left(\hat{E}-E_{n}\right) u_{n}^{2} \mathrm{~d} x=0 \text { and } \lim _{n \rightarrow \infty} \int_{\Omega}\left(\hat{E}-E_{n}\right) u^{2} \mathrm{~d} x=0 .
$$

The second limit in 30 follows from the weak convergence of $\left\{E_{n}\right\}$ in $L^{2}(D)$, since $u \in L^{\infty}(D)$. However, the verification of the first limit in (30) requires more work. To this end, let us recall that:

$$
\begin{cases}\epsilon^{2} \Delta u_{n}+u_{n}-u_{n}^{2}-E_{n}(x) u_{n}=0, & x \in \Omega, \\ u_{n}(x)=0, & x \in \partial \Omega .\end{cases}
$$


By multiplying both sides of (31) by $u_{n}$, and using the divergence theorem, we obtain:

$$
\begin{aligned}
\epsilon^{2} \int_{\Omega}\left|\nabla u_{n}\right|^{2} \mathrm{~d} x & =\int_{\Omega}\left(u_{n}^{2}-u_{n}^{3}-E_{n} u_{n}^{2}\right) \mathrm{d} x \\
\left(\text { as } u_{n} \geq 0, \text { and } E_{n} \geq 0\right) & \leq \int_{\Omega} u_{n}^{2} \mathrm{~d} x \\
\text { (by Proposition } 8 \text { ) } & \leq|\Omega| .
\end{aligned}
$$

Hence, $\left\{u_{n}\right\}$ is a bounded sequence in $H_{0}^{1}(\Omega)$. This, in turn, implies the existence of a subsequence of $\left\{u_{n}\right\}$ — still denoted $\left\{u_{n}\right\}$ - and $\tilde{u} \in H_{0}^{1}(\Omega)$, such that $u_{n} \rightarrow \tilde{u}$ in $H_{0}^{1}(\Omega)$, and $u_{n} \rightarrow \tilde{u}$ in $L^{2}(\Omega)$. Recalling that $0 \leq u_{n} \leq 1$, by extracting a further subsequence if necessary, it follows from Lebesgue dominated convergence theorem that $u_{n}^{2} \rightarrow \tilde{u}^{2}$ in $L^{2}(\Omega)$. Next, we write:

$$
\int_{D}\left(\hat{E}-E_{n}\right) u_{n}^{2} \mathrm{~d} x=\int_{D}\left(\hat{E}-E_{n}\right)\left(u_{n}^{2}-\tilde{u}^{2}\right) \mathrm{d} x+\int_{D}\left(\hat{E}-E_{n}\right) \tilde{u}^{2} \mathrm{~d} x
$$

Obviously, the right hand side of (32) tends to zero as $n$ goes to infinity. The proof of item (ip) is complete.

Before proving the last two properties, we claim that the operator $\mathcal{G}: \overline{\mathcal{R}} \rightarrow L^{2}(\Omega)$ defined by $\mathcal{G}(E):=u_{E}^{2}$ is continuous on $\overline{\mathcal{R}}$, and the set $B:=\{E \in \overline{\mathcal{R}}: \mathcal{G}(E)>0\}$ is open in $\overline{\mathcal{R}}$. We know from Proposition 8 that for every $E \in \overline{\mathcal{R}}, G(E)$ is either (strictly) positive or zero on $\Omega$. Recalling $[18$, we infer that $\Phi(E)=0$ if $u_{E}=0$, and $\Phi(E)<0$ if $u_{E}>0$. The openness of $B$ in $\overline{\mathcal{R}}$ follows by using the continuity of $\Phi$. To prove the continuity of $\mathcal{G}$, it suffices to show that $\tilde{u}=u_{\hat{E}}$. By (31) we have:

$$
\forall \phi \in C_{0}^{\infty}(\Omega): \epsilon^{2} \int_{\Omega} \nabla u_{n} \cdot \nabla \phi \mathrm{d} x+\int_{\Omega}\left(-u_{n} \phi+u_{n}^{2} \phi+E_{n} u_{n} \phi\right) \mathrm{d} x=0 .
$$

Since $u_{n} \rightarrow \tilde{u}$ in $H_{0}^{1}(\Omega), u_{n} \rightarrow \tilde{u}$ strongly in $L^{2}(\Omega)$, and $E_{n} \rightarrow \hat{E}$ in $L^{2}(\Omega)$, from 33 we obtain:

$$
\forall \phi \in C_{0}^{\infty}(\Omega): \epsilon^{2} \int_{\Omega} \nabla \tilde{u} \cdot \nabla \phi \mathrm{d} x+\int_{\Omega}\left(-\hat{u} \phi+\tilde{u}^{2} \phi+\hat{E} \tilde{u} \phi\right) \mathrm{d} x=0 .
$$

If $u_{\hat{E}}=0$, then it follows from Proposition 8 that $\tilde{u}=u_{\hat{E}}$. On the other hand, if $u_{\hat{E}}>0$, we need to show that $\tilde{u} \neq 0$. Let us pass to the limit in the following equation:

$$
\Phi\left(E_{n}\right)=\frac{\epsilon^{2}}{2} \int_{\Omega}\left|\nabla u_{n}\right|^{2} \mathrm{~d} x-\frac{1}{2} \int_{\Omega} u_{n}^{2} \mathrm{~d} x+\frac{1}{3} \int_{\Omega} u_{n}^{3} \mathrm{~d} x+\frac{1}{2} \int_{\Omega} E_{n} u_{n}^{2} \mathrm{~d} x,
$$

which leads to:

$$
0>\Phi(\hat{E})=\liminf _{n \rightarrow \infty} \Phi\left(E_{n}\right) \geq \frac{\epsilon^{2}}{2} \int_{\Omega}|\nabla \tilde{u}|^{2} \mathrm{~d} x-\frac{1}{2} \int_{\Omega} \tilde{u}^{2} \mathrm{~d} x+\frac{1}{3} \int_{\Omega} \tilde{u}^{3} \mathrm{~d} x+\frac{1}{2} \int_{\Omega} \hat{E} \tilde{u}^{2} \mathrm{~d} x
$$

This shows that $\tilde{u} \neq 0$, as desired.

(ii) Let us first prove that $\Phi$ is concave. Take $E_{1}, E_{2} \in \overline{\mathcal{R}}, t \in(0,1)$, and $E_{t}:=t E_{1}+(1-t) E_{2}$. For $v \in H_{0}^{1}(D)^{+}$, we have:

$$
\Phi\left(v, E_{t}\right)=t \Phi\left(v, E_{1}\right)+(1-t) \Phi\left(v, E_{2}\right),
$$

in which the bivariate variant $\Phi(.,$.$) is as in (20). By taking the infimum of both sides of (34) with$ respect to $v \in H_{0}^{1}(D)^{+}$, we obtain:

$$
\Phi\left(E_{t}\right) \geq t \Phi\left(E_{1}\right)+(1-t) \Phi\left(E_{2}\right),
$$


which proves the concavity of $\Phi$. We prove the second assertion by contradiction. To that end, by recalling the openness of $B$, assume that for some $E_{1} \in B \backslash\{E\}$ and $t \in(0,1)$, we have:

$$
\Phi\left(E_{t}\right)=t \Phi(E)+(1-t) \Phi\left(E_{1}\right) .
$$

From (35), and after some straightforward calculations using (21), we obtain:

$$
\Phi\left(u_{E}, E\right)=\Phi\left(u_{E_{t}}, E\right) \quad \text { and } \quad \Phi\left(u_{E_{1}}, E_{1}\right)=\Phi\left(u_{E_{t}}, E_{1}\right) .
$$

Once again, by using the minimality of $u_{E}$ and $u_{E_{1}}$ (Lemma 12), we obtain $u_{E}=u_{E_{t}}=u_{E_{1}}$. Thus, substituting in the main BVP (6), we have:

$$
\begin{cases}\epsilon^{2} \Delta u_{E}+u_{E}-u_{E}^{2}-E(x) u_{E}=0, & x \in \Omega, \\ \epsilon^{2} \Delta u_{E_{1}}+u_{E_{1}}-u_{E_{1}}^{2}-E_{1}(x) u_{E_{1}}=0, & x \in \Omega .\end{cases}
$$

This implies that $\left(E-E_{1}\right) u_{E}=0$, almost everywhere in $\Omega$. As $u_{E}$ is positive, then we must have $E=E_{1}$, almost everywhere in $\Omega$, which is a contradiction.

(iii) To derive formula (24), we first derive the following equation similar to 29]:

$$
\forall E_{1}, E_{2} \in \overline{\mathcal{R}}: \quad \frac{1}{2} \int_{\Omega}\left(E_{2}-E_{1}\right) u_{E_{2}}^{2} \mathrm{~d} x \leq \Phi\left(E_{2}\right)-\Phi\left(E_{1}\right) \leq \frac{1}{2} \int_{\Omega}\left(E_{2}-E_{1}\right) u_{E_{1}}^{2} \mathrm{~d} x .
$$

Now, by setting $E_{1}:=E$ and $E_{2}:=E+t v=E+t(F-E)$, from (36) we obtain:

$$
\frac{1}{2} \int_{\Omega} t v u_{E+t v}^{2} \mathrm{~d} x \leq \Phi(E+t v)-\Phi(E) \leq \frac{1}{2} \int_{\Omega} t v u_{E}^{2} \mathrm{~d} x
$$

which implies that:

$$
\frac{1}{2} \int_{\Omega} v u_{E+t v}^{2} \mathrm{~d} x \leq \frac{\Phi(E+t v)-\Phi(E)}{t} \leq \frac{1}{2} \int_{\Omega} v u_{E}^{2} \mathrm{~d} x
$$

as $t>0$. On the other hand, by applying the continuity of $\mathcal{G}$ we know that $\lim _{t \rightarrow 0^{+}} u_{E+t v}^{2}=u_{E}^{2}$ in $L^{2}(\Omega)$, which, combined with (37), implies that:

$$
\lim _{t \rightarrow 0^{+}} \frac{\Phi(E+t v)-\Phi(E)}{t}=\frac{1}{2} \int_{\Omega} v u_{E}^{2} \mathrm{~d} x
$$

as desired.

\section{Concluding remarks}

The theory behind optimization of convex functionals over rearrangement classes, as used in this paper, was originally laid out by Burton [Bur87] to study vortex rings, i. e., in the context of fluid dynamics. The abstract formulation of rearrangement optimization problems, however, suggests a greater potential for applications. In essence, one has a set of resources which should be deployed to solve a given problem, and the main task is to find the optimal arrangement/permutation of resources.

Since Burton's seminal work, there has been a steady flow of contribution to the theory and its applications in fluid mechanics [EM91; EN95], finance [EH09; Rüs13], free boundary problems [EM14], construction of robust membranes [Ema+17], and eigenvalue problems [EZR11], to name a few. In the current paper, we have extended this range of applications to include a theoretical result in marine economy. We showed that, using the theory of rearrangements, we can generalize the original results of Kurata and Shi [KS08], whilst keeping technicalities to a minimum, which is another witness to the power of the theory. Furthermore, we were able to devise a very straightforward efficient numerical algorithm for solving the minimization problem, which demonstrates that the theory can lend itself well to numerical computations. 


\section{References}

[Bur87] Burton, G. R. "Rearrangements of functions, maximization of convex functionals, and vortex rings". In: Math. Ann. 276.2 (1987), pp. 225-253.

[Bur89a] Burton, G. R. "Variational problems on classes of rearrangements and multiple configurations for steady vortices”. In: Ann. Inst. H. Poincaré Anal. Non Linéaire 6.4 (1989), pp. 295-319.

[Bur89b] Burton, G. "Rearrangements of functions, saddle points and uncountable families of steady configurations for a vortex". In: Acta Mathematica 163.1 (1989), pp. 291-309.

[CC89] Cantrell, R. S. and Cosner, C. "Diffusive logistic equations with indefinite weights: population models in disrupted environments". In: Proceedings of the Royal Society of Edinburgh: Section A Mathematics 112.3-4 (1989), pp. 293-318. Dor: 10.1017/S030821050001876X

[CCP13] Cosner, C., Cuccu, F., and Porru, G. "Optimization of the first eigenvalue of equations with indefinite weights". In: Adv. Nonlinear Stud. 13.1 (2013), pp. 79-95.

[EFL18] Emamizadeh, B., Farjudian, A., and Liu, Y. "Optimal Harvesting Strategy Based on Rearrangements of Functions". In: Applied Mathematics and Computation 320 (2018), pp. 677-690. DoI: 10.1016/j . amc.2017.10.006

[EFZR16] Emamizadeh, B., Farjudian, A., and Zivari-Rezapour, M. "Optimization Related to Some Nonlocal Problems of Kirchhoff Type". In: Canad. J. Math. 68.3 (2016), pp. 521-540. Dor: 10 . 4153/CJM-2015-040-9.

[EH09] Emamizadeh, B. and Hanai, M. A. "Rearrangements in real estate investments". In: Numerical Functional Analysis and Optimization 30.5-6 (2009), pp. 478-485.

[EL15] Emamizadeh, B. and Liu, Y. "Constrained and unconstrained rearrangement minimization problems related to the $p$-Laplace operator”. In: Israel J. Math. 206.1 (2015), pp. 281-298. Dor: 10.1007/s11856-014-1141-9.

[EM14] Emamizadeh, B. and Marras, M. "Rearrangement optimization problems with free boundary". In: Numer. Funct. Anal. Optim. 35.4 (2014), pp. 404-422.

[EM91] Elcrat, A. R. and Miller, K. G. "Rearrangements in steady vortex flows with circulation". In: Proc. Amer. Math. Soc. 111.4 (1991), pp. 1051-1055.

[Ema+17] Emamizadeh, B., Farjudian, A., Liu, Y., and Marras, M. "How to construct a robust membrane using multiple materials". Under review. 2017.

[EN95] Elcrat, A. and Nicolio, O. "An iteration for steady vortices in rearrangement classes". In: Nonlinear Anal. 24.3 (1995), pp. 419-432.

[ET88] Eydeland, A. and Turkington, B. "A Computational Method of Solving Free-boundary Problems in Vortex Dynamics”. In: J. Comput. Phys. 78.1 (1988), pp. 194-214. Dor: 10.1016/ 0021-9991(88)90044-7

[Eva10] Evans, L. C. Partial differential equations. 2nd. Graduate Studies in Mathematics. American Mathematical Society, 2010.

[EZR11] Emamizadeh, B. and Zivari-Rezapour, M. "Rearrangements and minimization of the principal eigenvalue of a nonlinear Steklov problem”. In: Nonlinear Anal. 74.16 (2011), pp. 5697-5704.

[Fra00] Fraenkel, L. E. An introduction to maximum principles and symmetry in elliptic problems. Vol. 128. Cambridge Tracts in Mathematics. Cambridge: Cambridge University Press, 2000.

[KS08] Kurata, K. and Shi, J. “Optimal spatial harvesting strategy and symmetry-breaking”. In: Applied Mathematics and Optimization 58.1 (2008), pp. 89-110.

[LE16] Liu, Y. and Emamizadeh, B. "Rearrangement minimization problems with indefinite external forces”. In: Nonlinear Analysis: Theory, Methods $\mathcal{E}$ Applications 145 (2016), pp. 162-175. Dor: http://dx.doi.org/10.1016/j.na.2016.08.006 
[Mur02] Murray, J. D. Mathematical Biology: I. An Introduction. 3rd ed. Springer, 2002.

[OSS02] Oruganti, S., Shi, J., and Shivaji, R. "Diffusive logistic equation with constant yield harvesting, I: Steady States”. In: Trans. Amer. Math. Soc. 354.9 (2002), pp. 3601-3619. Dor: 10 . 1090/ S0002-9947-02-03005-2.

[Rüs13] Rüschendorf, L. Mathematical Risk Analysis: Dependence, Risk Bounds, Optimal Allocations and Portfolios. Springer, 2013. 